\title{
Mott Polarimeter Upgrade at Jefferson Lab
}

\author{
J. Grames, ${ }^{a}$ A. K. Opper, ${ }^{b}$ M. J. McHugh, ${ }^{* b}$ M. Poelker, ${ }^{a}$ C. Sinclair, ${ }^{c}$ and R. \\ Suleiman ${ }^{a}$ \\ ${ }^{a}$ Jefferson Lab \\ ${ }^{b}$ The George Washington University \\ ${ }^{c}$ Cornell University \\ Email: mjmchughegwu.edu
}

\begin{abstract}
A Mott polarimeter with a design optimized for $5.5 \mathrm{MeV} / \mathrm{c}$ has been in routine use at the CEBAF accelerator for well over a decade, providing polarization measurements approaching $1 \%$ accuracy. Measurements with different target materials ( $\mathrm{Au}, \mathrm{Ag}, \mathrm{Cu}$ ) over a range of target thicknesses $(100-10,000 \AA)$, and beam energies between 2 and $8 \mathrm{MeV}$ allow us to determine the effective analyzing power with a high degree of certainty. Recent and planned improvements in our polarimeter configuration, detectors and data acquisition system, coupled with a low 31 $\mathrm{MHz}$ repetition rate beam allow us to distinguish and suppress electrons that do not originate from the target foil. This work coupled with a significant effort to produce a detailed GEANT4 model of the polarimeter is part of an effort to determine systematic uncertainties at the level of the theoretically calculated analyzing power. We describe our activities and a series of planned measurements that will allow us to demonstrate and possibly improve the precision and accuracy of polarization measurements at JLab, as required for future parity violation experiments.
\end{abstract}

XVth International Workshop on Polarized Sources, Targets, and Polarimetry,

September 9-13, 2013

Charlottesville, Virginia, USA

\footnotetext{
${ }^{*}$ Speaker.
} 


\section{Introduction}

The MeV Mott Polarimeter is located in the Continuous Electron Beam Accelerator Facility (CEBAF) injector at Jefferson Lab (JLab). It is used to measure the transverse polarization of the electron beam in the $2-8 \mathrm{MeV}$ energy range. The polarimeter (Fig. 四) measures the elastic scattering asymmetry of electrons incident on the nuclei of a thin target foil. The foils used include gold, silver, and copper and range in thickness from 100-10,000 $\AA$. This polarimeter is used in conjunction with the spin rotators to set the polarization received by the experimental halls.

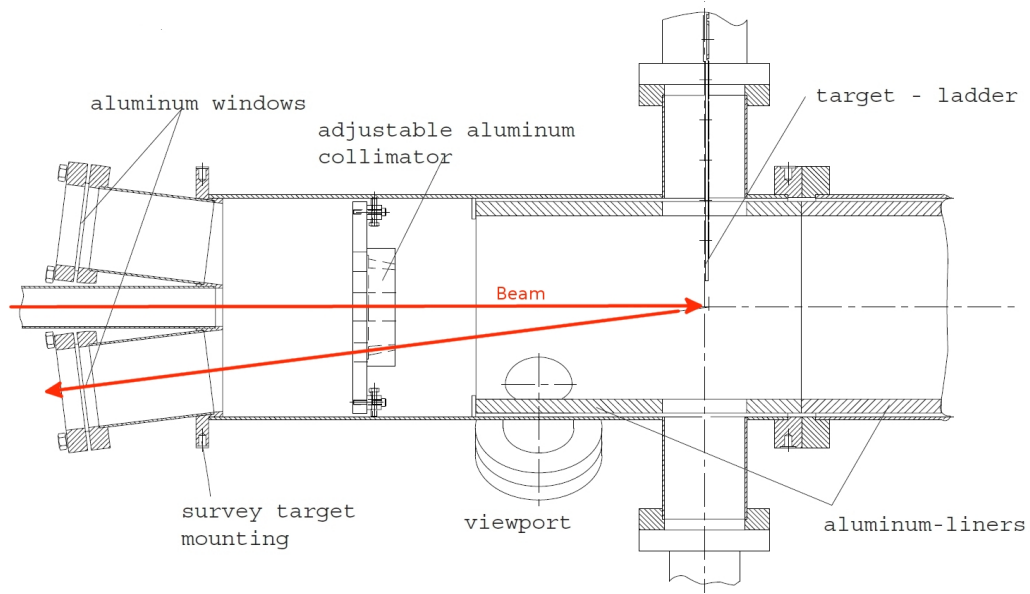

Figure 1: Cross-section of the polarimeter's scattering chamber. A typical event is shown in red.

The elastically scattered electrons from the target foil pass through an aluminum collimator which sets the scattering angle of $172.6^{\circ} \pm 0.1^{\circ}$ with a per quadrant solid angle of $0.18 \mathrm{msr}$. The scattered electrons pass through the collimator, then pass through the $0.05 \mathrm{~mm}$ thick aluminum window and into the detector packages. Each detector package contains two plastic scintillators connected to PMTs for readout: a $1 \mathrm{~mm} \times 25.4 \mathrm{~mm} \times 25.4 \mathrm{~mm}$ wafer scintillator, the $\Delta$ E detector, and a cylindrical $76.2 \mathrm{~mm}$ diameter, $63.5 \mathrm{~mm}$ long scintillator, the E detector, which functions as a stop detector and calorimeter with a $3 \%$ energy resolution. The data acquisition system utilizes a coincidence trigger on the $\mathrm{E}$ and $\Delta \mathrm{E}$ detectors to filter out any incident neutral particles. The resulting spectrum (Fig. D) contains a strong elastic signal from the target as well, background from other beam line elements, primarily the aluminum dump plate, and the "off-peak" elastic events (events down to $80 \%$ of the elastic peak energy, which are neither exclusively elastic or background). Flipping the beam polarization at a rate of $30 \mathrm{~Hz}$ allows us to calculate the total polarization using the cross-ratio method described in [W]. We find the Mott scattering asymmetry to be:

$$
A_{U D}=\frac{1-\sqrt{N_{U}^{\uparrow} N_{D}^{\downarrow} / N_{U}^{\downarrow} N_{D}^{\uparrow}}}{1+\sqrt{N_{U}^{\uparrow} N_{D}^{\downarrow} / N_{U}^{\downarrow} N_{D}^{\uparrow}}}
$$

This asymmetry is proportional to the polarization as shown here:

$$
A_{U D}(\theta)=S(\theta) P_{x}
$$


where $S(\theta)$, known as the Sherman function, is the analyzing power [ [ $]$ ]. Similar ratios are constructed to measure the vertical polarization as well. Using this method, the asymmetry is measured with $0.5 \%$ statistical precision in about 5 minutes using a $1 \mu \mathrm{m}$ Au foil and $1 \mu \mathrm{A}$ of beam current.

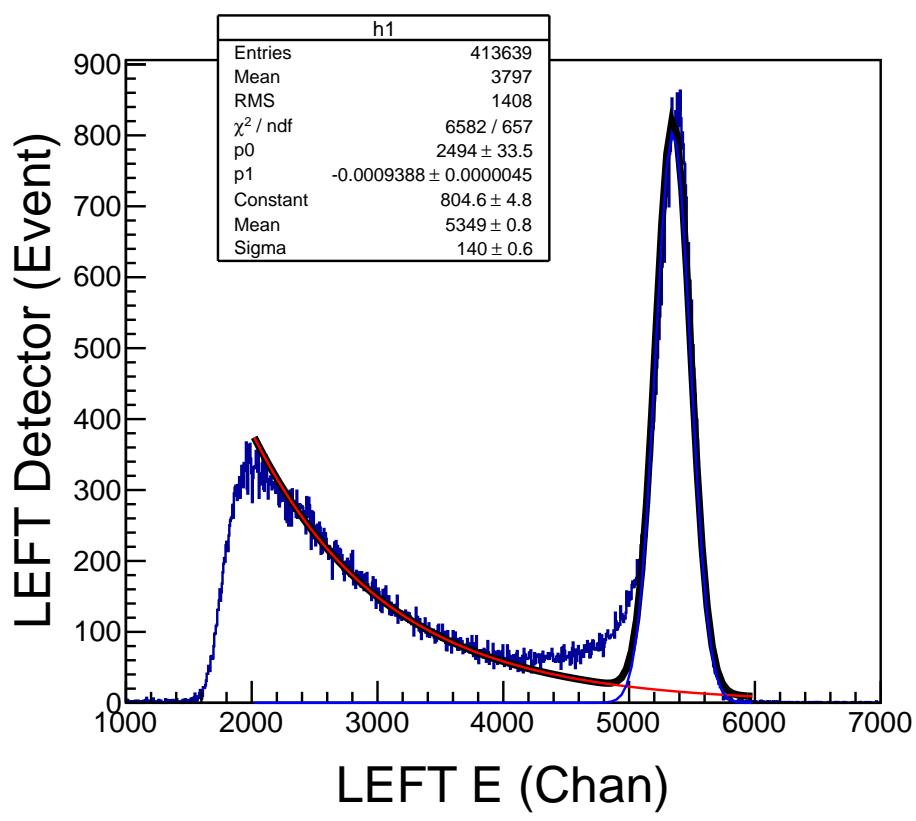

Figure 2: Spectrum seen in the beam left detector package during a $5 \mathrm{MeV}$ run on $1 \mu \mathrm{m}$ Au foil.

When this polarimeter was commissioned over a decade ago, the total systematic uncertainty was quoted to be $1.1 \%$, dominated by the uncertainty in the theoretical uncertainty in the Sherman function [B]. At this time, the polarimeters in the experimental halls were only precise to the $5 \%$ level and therefore all polarization measurements were found to be in agreement. Over the lifetime of the Mott polarimeter, the polarization level of the CEBAF beam has gone from $<50 \%$ to almost 90\%. In addition, the current precision of the hall polarimeters has approached the $1 \%$ level. At this precision and assuming the quoted Mott polarimeter accuracy above, a measured polarization discrepancy of 2-3 $\sigma$ has been observed. This discrepancy motivates the current effort to quantify and reduce the systematic errors on the Mott polarimeter.

The current effort involves several avenues which are being pursued simultaneously. There will be work on the theory side to determine the precision of the single nucleus Sherman function. This effort will be supported by additional measurements on a larger number of target materials (including lower $\mathrm{Z}$ nuclei such as aluminum) to determine and limit the size of radiative contributions to the uncertainty. The updated theory will be placed into a GEANT4 [䧃] simulation of the polarimeter which will be used to benchmark the extrapolation of the effective Sherman function to zero target thickness (a source of additional uncertainty). This GEANT4 simulation will also be used to reproduce some of the observed detector phenomena. At the same time, there is work being done to improve the apparatus itself to ensure the cleanest possible signal. This includes changing beam bunch rate to isolate target events and designing and building a new beryllium dump plate to reduce back-scatter. 


\section{Detector Response Simulation}

Typical running conditions during the past decade had very few controls in place to reduce the amount of background. To reduce the impact of this background, stringent energy cuts were applied, which removed a large portion of elastically scattered electrons from the asymmetry calculation as a side-effect. An early goal of the simulation effort was to determine the source of the off-peak events which carry a large Mott asymmetry. An example of this phenomena is shown in Fig. [].

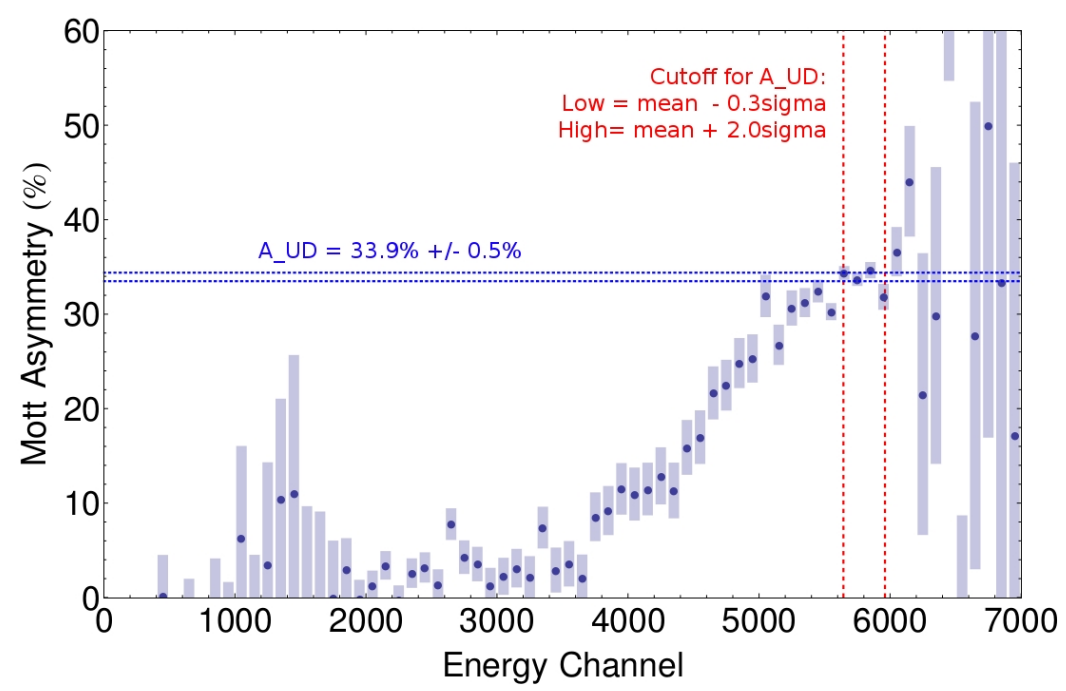

Figure 3: Mott asymmetry as a function of energy. The data outside of the dotted lines are excluded from the asymmetry calculation.

Simulations performed to this point indicate that the off-peak events which carry a reduced asymmetry consist largely of elastically scattered electrons which lose energy in the collimators and aluminum detector windows. The dilution of this asymmetry is due to beamline backgrounds which carry no physics asymmetry. These results can be observed when comparing simulations without beamline elements (collimator, windows, dump etc.) to those with these beamline elements and to actual data with background subtracted (Fig. 团). The simulation and data agree particularly well in the 3.8-4.5 MeV range, which is exactly the range of interest. More refined simulations will be developed and data will be taken to numerically confirm this hypothesis and to provide insight for new energy cuts and background dilution factors that need to be included in the asymmetry calculations.

\section{Background Reduction}

The second avenue of improvements being pursued include work to better quantify and reduce backgrounds that are currently found in the data. These background events are largely due to back-scattered electrons from the dump plate with a small amount coming from the scattering chamber walls and other beamline elements. The background has zero physics asymmetry. For thinner targets, this background can account for as much as $4 \%$ of the events included in the asymmetry calculation. The two main methods of background reduction being pursued are time-of-flight 


\section{E Spectra}

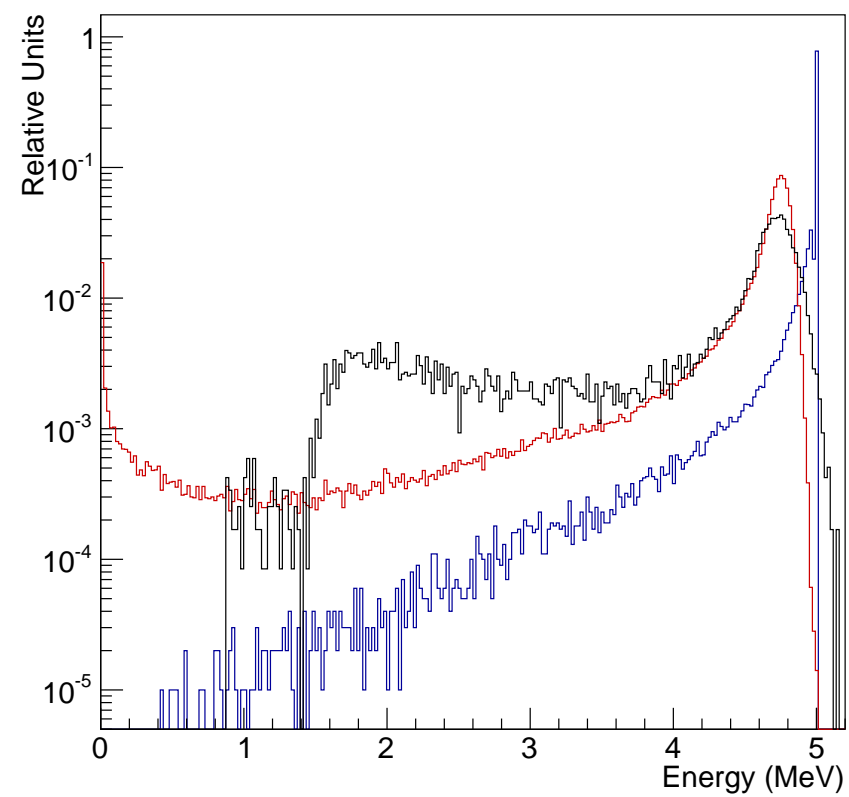

Figure 4: A comparison of spectra from: simulation with no beamline elements except the target and detector packages (blue), a simulation with all beamline elements (red), and actual data with backgrounds separated by a timing cut (black). All histograms are normalized to have unit integrals. Events in simulation are generated at the target scattering vertex.

exclusions and a redesigned beam dump to reduce back-scatter. During previous running, the repetition rate of beam bunches was $499 \mathrm{MHz}$. In this configuration, electrons from the dump would arrive at the detectors at the same time as good events. However, the injector can now produce lower bunch rates cleanly. Running at $31 \mathrm{MHz}$ allows easy isolation of events that arrive in time with electrons from the target (Fig. [). Using this method drastically reduces the background event count in asymmetry calculations.

In addition, a beryllium dump plate will replace the current aluminum plate dump. The current dump plate is simply a $25.4 \mathrm{~mm}$ thick aluminum end flange attached to the vacuum chamber of the polarimeter. During runs on $1 \mu \mathrm{m}$ Au foil, this dump produces roughly the same event rate as the target. The new dump plate, designed with the help of GEANT4 simulation, will consist of $6.35 \mathrm{~mm}$ of beryllium backed by $19.05 \mathrm{~mm}$ of water-cooled copper. Simulation results are shown in Fig. 6 which predict a factor of 4 suppression of back-scattered electrons when using the new dump. Additionally, thermal calculations indicate that this new dump can handle much higher beam currents, allowing for faster measurements.

\section{Summary}

The Mott polarimeter has been in service for almost two decades at JLab. There is now an effort being made to quantify properly and reduce the systematic uncertainty of this polarimeter. The effort is focused in three areas. Firstly, theoretical uncertainties will be experimentally limited 

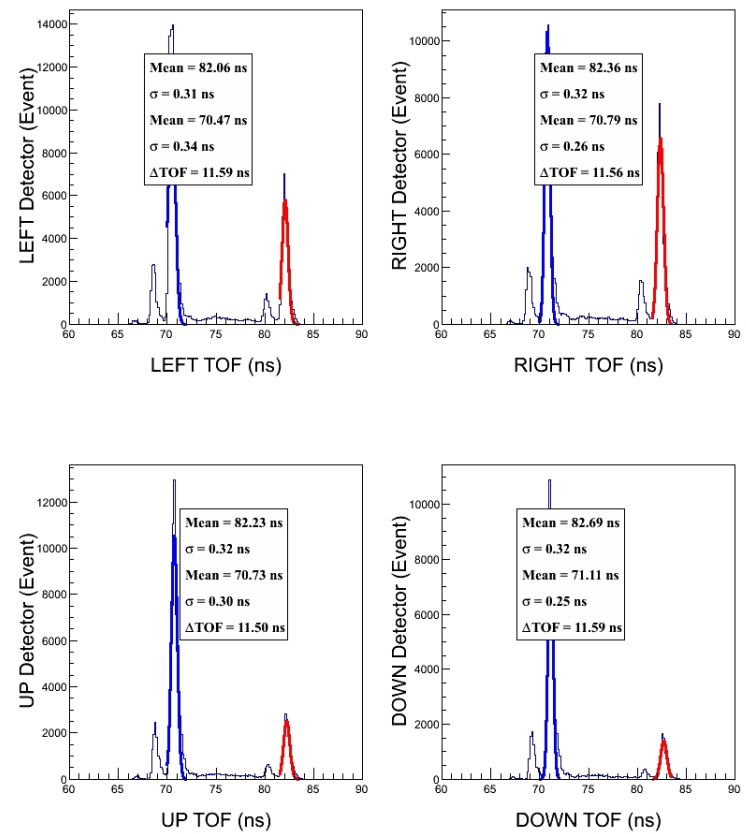

Figure 5: Time of flight separation using $31 \mathrm{MHz}$ bunch rate. Blue are from the target, red are from the dump.

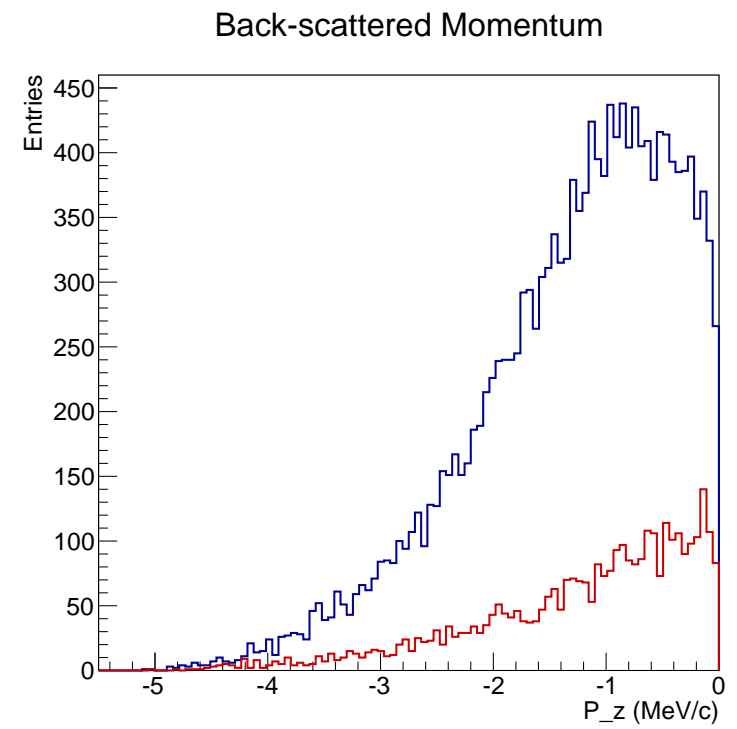

Figure 6: Comparison of back-scattered momentum spectra from the $\mathrm{Al}$ dump and $\mathrm{BeCu}$ dump.

using a series of targets varying widely in $\mathrm{Z}$ and thickness. Secondly, the improved theory will be incorporated into GEANT4 simulations in order to benchmark the experimental results and reduce uncertainties resulting from target-thickness extrapolation of the Sherman function. Thirdly, improved data taking methods and polarimeter design will be used to reduce the amount of background events observed. These changes are all being guided by and incorporated into an ongoing 
effort to simulate and document the Mott polarimeter accurately for future use as an additional absolute polarimeter in JLab operations and experiments. Work done thus far includes testing the new bunch rate and event exclusion based on timing data, design and simulation of a beryllium dump plate, and simulation of the detector response to elastic electrons to determine the source of off-peak elastic events.

\section{References}

[1] G. G. Ohlsen, Jr. and P. W. Keaton, Nucl. Instrum. Meth. 109, 41 (1973)

[2] T. J. Gay and F. B. Dunning, Rev. Sci. Instrum. 63, 1635 (1992); doi: 10.1063/1.1143371

[3] M. Steigerwald AIP Conf. Proc. 570 935-942 (2000)

[4] S. Agostinelli et al., GEANT4 - a simulation toolkit, Nucl. Instr. Meth. A, 506, no. 3, pp. 250-303, (2003). 